\title{
Sistema Unificado de Consulta e Análise da Acidentalidade em Rodovias
}

\author{
Federais de Pernambuco (SUCAARF-PE) \\ Unified System of Consultation and Analysis of Accidentality in Federal Roads of \\ Pernambuco (SUCAARF-PE) \\ Vinícius Henrique de Mendonça ${ }^{1}$, Thalles Vitelli Garcez ${ }^{* 1}$ \\ ${ }^{1}$ Universidade Federal de Pernambuco, Pernambuco, Brasil.
}

\begin{tabular}{|c|c|}
\hline I NFO ARTIGO & RES U MO \\
\hline $\begin{array}{l}\text { Palavras-chave: } \\
\text { Acidentes de trânsito, } \\
\text { Decisão Multicritério, } \\
\text { Rodovias Federais, } \\
\text { Sistema de Apoio à } \\
\text { Decisão, } \\
\text { Pernambuco. }\end{array}$ & $\begin{array}{l}\text { O trabalho apresenta a concepção, modelagem e disponibilidade de um } \\
\text { Sistema Unificado de Consulta e Análise da Acidentalidade em Rodovias } \\
\text { Federias de Pernambuco, SUCAARF-PE, aplicado à avaliação e tomada de } \\
\text { decisão multicritério para priorização trechos rodovias federais de } \\
\text { Pernambuco em hierarquia de criticidade da acidentalidade de trânsito, } \\
\text { através do uso dos dados disponíveis no banco de dados da Polícia Rodoviária } \\
\text { Federal (PRF). Para esse desenvolvimento, foi utilizada a combinação de } \\
\text { técnicas de desenvolvimento de banco de dados interativo, métodos } \\
\text { matemáticos para o desenvolvimento de um modelo de apoio à decisão } \\
\text { multicritério, juntamente com a linguagem de programação Java para geração } \\
\text { da interface gráfica do sistema e disponibilização do aplicativo para o usuário } \\
\text { nas lojas Android. }\end{array}$ \\
\hline ARTICLE INFO & A B S T R A C T \\
\hline $\begin{array}{l}\text { Keywords: } \\
\text { Decision Support System, } \\
\text { Federal Roads, } \\
\text { Multicriteria Decision, } \\
\text { Pernambuco, } \\
\text { Traffic-accidents. }\end{array}$ & $\begin{array}{l}\text { The paper presents the conception, modeling and availability of a Unified } \\
\text { System of Consultation and Analysis of Accidentality in Federal Roads of } \\
\text { Pernambuco, SUCAARF-PE, applied to the evaluation and decision-making } \\
\text { of multicriteria to prioritize sections of federal roads in Pernambuco in a } \\
\text { hierarchy of criticality of accidentality traffic, using the data available in the } \\
\text { Federal Road Police (PRF) database. For this development, it was used the } \\
\text { combination of interactive database development techniques, mathematical } \\
\text { methods for the development of a multicriteria decision support model, } \\
\text { together with the Java programming language for generating the system's } \\
\text { graphical interface and making the application available for the user in } \\
\text { Android stores. }\end{array}$ \\
\hline
\end{tabular}

\section{Introdução}

O número de acidentes de trânsito nas rodovias brasileiras é ainda alto. Segundo dados da Organização Mundial

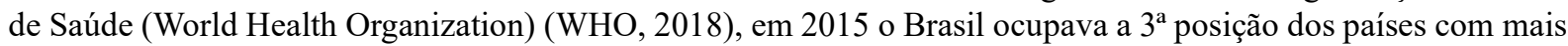
mortes no trânsito em termos de valor absoluto, atrás apenas da Índia e China. Quando levado em consideração a razão do número de mortes pelo número total de habitantes, o Brasil ocupava a $14^{\mathrm{a}}$ posição, tendo em média 19.7 mortes por 100.000 habitantes, no mesmo ano de 2015.

Os números de acidentes no que se refere ao estado de Pernambuco não são menos alarmantes, já que, ocorreram por volta de 3.600 mortes de um total aproximado de 57 mil acidentes entre os anos de 2007 a agosto de 2015,

\footnotetext{
${ }^{*}$ Correspondência para autor: vinicius.mendonca@ufpe.br (de Mendonça, V.H.), thalles.garcez@ufpe.br (Garcez, T.V.) (ORCID: 0000-0001-8155-1768).
} 
com média de 6.712 acidentes por ano, sendo a causa "falta de atenção" a principal causa de acidentes (MARTINS; GARCEZ, 2017).

Os registros de acidentes de trânsito das rodovias federais do Brasil estão armazenados nos bancos de dados disponibilizados pela Policia Rodoviária Federal (PRF), que são de livre acesso (Dados Abertos) e estão separados por ano (de 2007 até 2020). Neste banco de dados, está contido fatos sobre as ocorrências e as pessoas envolvidas nos acidentes, tais como: causa e tipo do acidente, idade e sexo do envolvido, dentre outros aspectos relacionados às rodovias em que o acidente ocorreu e aos veículos envolvidos.

Assim, visto a grande quantidade de ocorrências e pessoas envolvidas em acidente, têm-se como resultado um banco de dados contendo milhares de registros sobre as ocorrências de acidente ou as pessoas envolvidas nos acidentes. Além disso, os dados contidos em seu banco de dados apresentam-se de forma crua e estática, necessitando-se transforma-las em informações através de sua organização, tratamento e refinação. Portanto, significados e finalidades conjuntas, do que os fatos individuais encontrados, podem ser gerados, facilitando assim a tomada de decisão presente ou futura (DE ALMEIDA; RAMOS, 2002; STAIR; REYNOLDS; DA SILVA, 2011).

Dessa forma, verificando-se o grande número de dados acerca das acidentalidades nas rodovias federais brasileiras, faz-se necessário um sistema de informação (SI), capaz de captar, gerenciar e disponibilizar organizadamente os dados, para que seja possível a realização do processamento em informações úteis e proporcionar auxílio aos interessados (população, governo e empresas), facilitando assim o acesso as informações e, subsidiar a tomada de decisão que venham a, reduzir os riscos de acidentes nas rodovias e realização de ações preventivas de redução dos acidentes.

Entre os vários tipos de sistemas de informação utilizados pelas organizações, se encontram os Sistemas de Apoio à Decisão (SAD), que além de incorporar um sistema de informação, são sistemas utilizados no processo decisório que proporcionam ao decisor acesso fácil à banco de dados e banco modelos, apoiando a tomada de decisão semiestruturada ou não-estruturada (SPRAGUE; WATSON, 1991).

O SAD proposto neste trabalho será nomeado de Sistema Unificado de Consulta e Análise da Acidentalidade em Rodovias Federias de Pernambuco (SUCAARF-PE). Ele foi desenvolvido com o objetivo principal de reunir, gerenciar e transformar o grande número de dados presentes nos bancos de dados de acidentes da PRF, possibilitar a geração de conhecimento e disponibilizar eficientemente as informações aos usuários de rodovias federais, como condutores, motoristas profissionais, caminhoneiros, turistas, etc.; que representam os principais agentes diretos e beneficiados pela implementação de um aplicativo dos trechos das rodovias neste contexto de análise.

Além disso, a utilização do SUCAARF-PE permite, através de seu banco de modelos, a capacidade de auxiliar o processo de análise e tomada de decisão destes usuários, com o objetivo de priorização dos trechos das rodovias sob a visão da acidentalidade, considerando a estrutura de preferência deste decisor (usuário), agregando múltiplos critérios através da abordagem de apoio à decisão multicritério.

Dessa forma, o aplicativo proposto torna-se uma ferramenta útil aos motoristas (profissionais ou viajantes), que utilizam as vias federais e necessitam analisar e determinar sua rota dentre um conjunto de trechos possíveis, que resulte uma menor propensão ao risco de acidentes na rodovia. Ainda, permite ao usuário visualizar o ranking em criticidade da acidentalidade dos trechos de rodovias analisadas considerando o perfil do condutor de forma a facilitar ao usuário direcionar esforços de ações preventivas à acidentes de trânsito. Por exemplo, se um determinado trecho é mais o crítico e sua principal causa de acidentes é causada por animais na pista, então o condutor deve realizar ações para redução da velocidade e deter maior atenção à pista.

\section{Referencial Teórico}

Para que o SAD auxilie no processo de tomada de decisão é necessário ser composto de um banco de dados, um banco de modelos e uma interface de interação entre o usuário e o sistema. Para Sprague e Watson (1991), um banco de dados é uma coleção de dados armazenados e organizados em um só local que permite compartilhamento e inter-relacionamento por múltiplos usuários.

Já um banco de modelos consiste em conjuntos de modelos matemáticos e estatísticos, os quais, juntos aos dados contidos no banco de dados, permitem que sejam feitos os mais variados tipos de análises através de simulações, resoluções de problemas, entre outros, gerando informações para auxiliar o decisor na tomada de decisão (DE ALMEIDA; RAMOS, 2002).

Por fim, sendo o componente mais importante do sistema para o usuário, a interface é o subsistema que prover 
diferentes e amigáveis diálogos entre o usuário e o sistema, habilitando o usuário a interagir com o SAD (SHIM, 2002). Para Rathnam e Mannino (1995), a interface de um sistema de apoio à decisão devem envolver displays, botões de comando de múltiplos controles, devendo ser exaustivamente explicado aos usuários para que não exista resistência de utilização do sistema. Segundo Pearson e Shim (1994), essa necessidade de tornar amigável a interface do sistema decorre sobretudo de atributos psicológicos do usuário, como preferências e comodidade, onde uma interface de difícil manipulação normalmente representará um obstáculo ao uso e provável fracasso do SAD.

Ademais, o SAD, por ser um sistema orientado a problemas não-estruturados ou semiestruturados, apresenta uma metodologia de desenvolvimento baseada na abordagem evolutiva. De acordo com essa abordagem, os SADs devem estar sempre evoluindo, sendo disponibilizadas atualizações e melhorias, visando agregar novos recursos, de forma a suportar de forma mais eficaz o processo decisório.

Dessa forma, o output fornecido pelo SAD pode tornar-se entradas para novas pesquisas, que implementem novas etapas e proporcionem uma otimização nos recursos pré-existentes e os inseridos pelas novas atualizações.

\section{Trabalhos Relacionados}

No que se refere as acidentalidades nas rodovias federais pernambucanas, Martins e Garcez (2019) desenvolvem um SI utilizando a linguagem de programação Visual Basic for Applications (VBA), realizando um estudo de análise e estatística descritiva dos panoramas dos acidentes de trânsito. Entretanto, o presente trabalho busca diferenciar-se dos trabalhos anteriores, disponibilizando o sistema desenvolvido para utilização pela sociedade e instituições interessada em seus resultados, como também permitir análise decisória para o processo de tomada de decisão.

Trabalos relacionados na proposição de um SAD no contexto de acidentes em rodovias federais são abrangentes, como o trabalho de Ryder et al. (2017), que se baseou no desenvolvimento de um SAD para os veículos com o de fornecer avisos de pontos críticos de acidentes aos motoristasm com base em análises na localização do veículo aplicado a um conjunto de dados históricos nacionais de acidentes. Verificou-se que, os avisos disponibilizados no veículo, em um teste de campo com 57 motoristas profissionais, durante um período de quatro semanas, apresentaram uma melhoria significativa no comportamento do motorista ao longo do tempo.

Martins et al. (2020) propuseram um modelo de decisão multicritério para priorizar trechos de estradas, com base em sua criticidade e nos riscos que os usuários enfrentam. O modelo foi aplicado pelo método FITradeoff porposto por de Almeida et al. (2016), para a problemática de ordenamento (FREJ; DE ALMEIDA; COSTA, 2019), devido à sua flexibilidade e por exigir menos esforço cognitivo do tomador de decisão para fornecer informações sobre suas preferências.

\section{Desenvolvimento do SAD SUCAARF-PE}

Para a construção do SAD foi utilizado à linguagem de programação Java e a plataforma Android Studio, de modo a criar as janelas e interface do sistema e suas funções lógicas, permitindo um desenvolvimento em formato de aplicativo para smartphone Android, e as informações sejam mostradas com maior facilidade de acordo com o que for requisitado pelo usuário. Além disso, o banco de dados da PRF sendo disponibilizado em formato de planilhas. No entanto, necessitou-se o desenvolvimento de um banco de dados próprio para o SAD, onde os dados antes presentes no banco da PRF, são tratados e alimentados ao sistema, facilitando assim que não seja necessário um acesso direto ao banco de dados da PRF.

O esquema proposto para criação do SAD é mostado na Figura 1, na qual é formado pelo banco de dados que se comunica com a interface do usuário e o banco de modelos do sistema, e sendo este responsável pela disponibilização da informação requisitada, e por fim, a interface do usuário, onde o usuário requisita às informações que necessita, e interage com o sistema para visualizar a saída. 


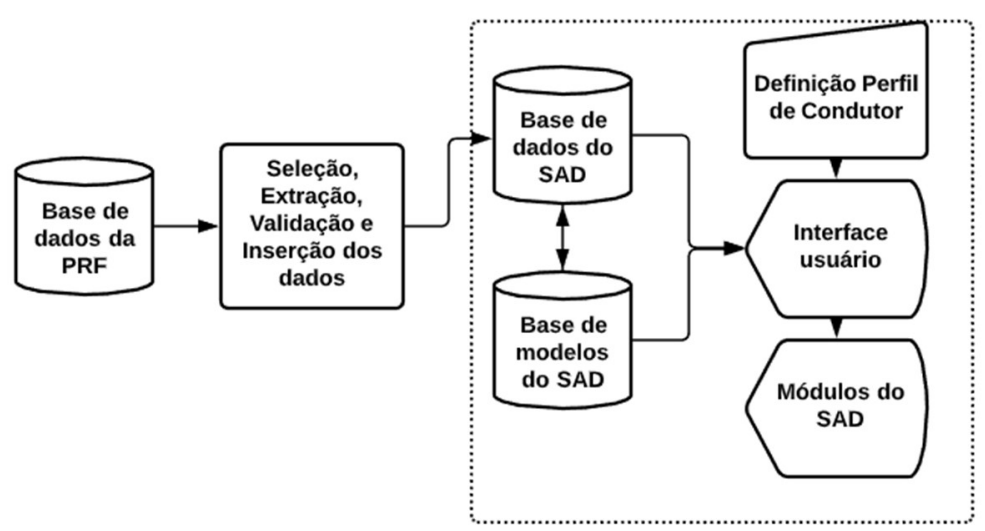

Figura 1 - Modelagem para o SAD SUCAARF-PE.

Primeiramente, para a construção do subsistema banco de dados do SUCAARF-PE, tem-se a seleção dos dados encontrados nos Dados Abertos da PRF, optando-se pelos anos que oferecesse um conjunto mais abrangente de informações e serem estatisticamente representativas da acidentalidade atual pernambucana, definindo assim, os anos de 2017 a agosto de 2019. Posteriormente, realizou-se o tratamento dos dados extraídos, retendo as ocorrências de trânsito no estado de Pernambuco, como também a sua respectiva validação, identificando possíveis erros, dados faltosos e incoerências, para posterior inserção no banco de dados do SUCAARF-PE.

Como resultado dos subsistemas banco de dados e interface usuário, têm-se, o mecanismo de consulta e extração de dados do banco de dados com o objetivo de oferecer uma análise descritiva dos dados, através de informações gráficas e tabelas de leituras, a partir de um perfil de análise selecionado pelo usuário.

Por fim, têm-se o subsistema do banco de modelos, que é composto por um modelo de decisão multicritério para o ranqueamento em criticidade da acidentalidade dos trechos, onde com os trechos das rodovias federais de Pernambuco definidos pelo usuário, o modelo através da avaliação multicritério e a estrutura de preferência do usuário (decisor), é possível determinar, por exemplo, os trechos que o usuário (motorista) necessita demandar maior atenção durante o percurso do trajeto.

\subsection{Modelagem do SAD SUCAARF-PE}

No momento que o usuário informa os dados de entrada do perfil condutor na interface de Definição do Perfil, o usuário é previamente direcionado a escolher à página de acesso que deseja navegar, que neste aplicativo é apresentada pela interface de Visualização Gráfica e a interface do Modelo Decisório, sendo estas os outputs do SAD. O esquema dos modelos compostos no SAD SUCAARF-PE proposto é mostrado na Figura 2.

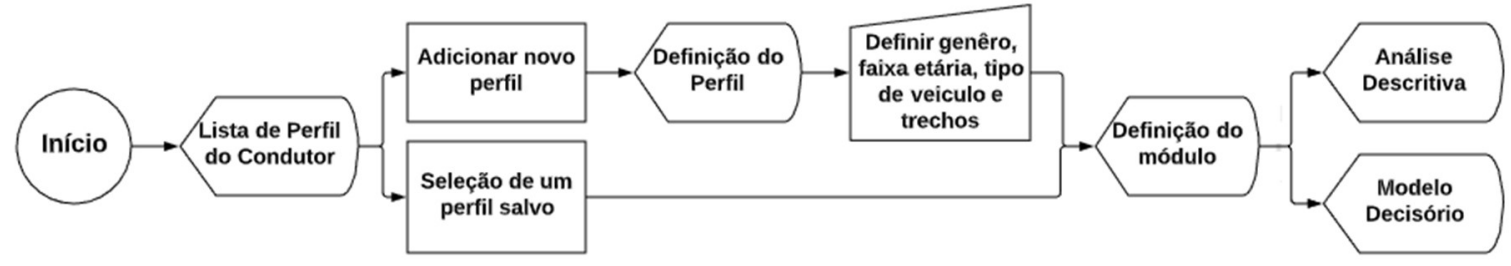

Figura 2 - Módulos do SAD SUCAARF-PE.

Primeiramente, o usuário tem como opção utilizar perfis salvos anteriormente para facilitar a utilização dos dados já preenchidos do perfil do condutor. Assim, caso o usuário (motorista) realize um mesmo trajeto rotineiramente, o aplicativo disponibiliza salvo em seu celular (smartphone) os dados do perfil do condutor preenchido previamente (vide Figura 3a).

Caso o usuário necessite preencher um novo perfil do condutor, ele seleciona a opção de "Adicionar novo perfil", 
ao qual será redirecionado a interface de Definição do Perfil onde serão encontrados espaços os quais devem ser selecionados pelo usuário de acordo com seu perfil e necessidades, como demonstrado na Figura 3b. Vale a ressalva que todas as opções possíveis já estão listadas, bastando, assim, apenas o usuário selecionar uma delas, como demonstrado na Figura 3c. Com isso, os dados desse perfil do condutor serão salvos no dispositivo do usuário e poderão ser utilizados posteriormente.
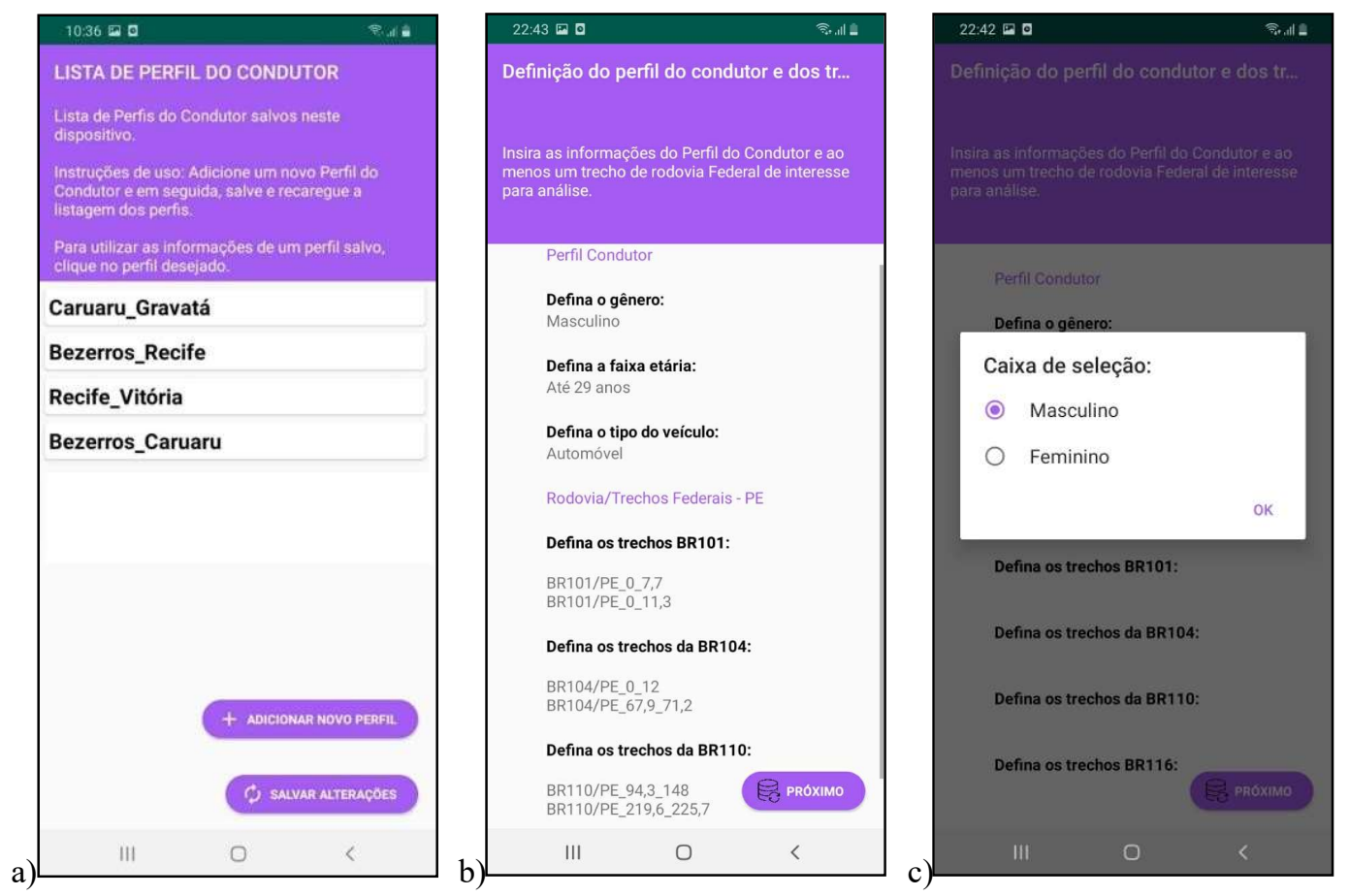

Figura 3 - Interfaces de entrada de dados do SAD SUCAARF-PE: a) Lista de perfis de condutor já salvos anteriormente; b) Definição de um novo condutor; c) Opções de seleção das características do perfil.

\subsection{Módulo de Análise Descritiva}

O SUCAARF-PE apresenta dois módulos principais (conforme mostrado na Figura 4a): i) módulo da análise descritiva do perfil do condutor, do veículo e do trecho da rodovia escolhidos; e, ii) Módulo Decisório: Priorização dos trechos de rodovias selecionadas.

A primeira saída ('Módulo da análise descritiva do perfil do condutor, do veículo e do trecho da rodovia escolhidos') apresenta uma análise descritiva dos dados obtidos na consulta ao banco de dados do SAD em formato de gráficos e tabelas, onde é proporcionado seis módulos de análise para o usuário, cada módulo analisando um conjunto distinto dos dados definidos pelo usuário.

O esquema do módulo de análise descritiva é mostrado na Figura 5, na qual, após o usuário determinar a opção de análise desejada, dentre as disponibilizadas, o banco de dados do SUCAARF-PE é consultado e são obtidos os dados compatíveis com as informações do perfil do condutor selecionado ou determinado pelo usuário anteriormente.

Primeiramente, os resultados apresentados pelo 'Módulo de Análise Descritiva' são representados por um menu no qual o usuário pode escolher as análises que desejar ver, conforme apresenta a Figura $4 \mathrm{~b}$. O usuário possui escolha entre seis opções:

1. CONDUTOR(ES): analisar os dados referentes a seu perfil do condutor (genêro e faixa etária), independente do trecho e tipo de veículo escolhido;

2. VEÍCULO(S): analisar os dados especificos para o tipo de veículo escolhido, independente do perfil do condutor e trecho(s) escolhido(s);

3. TRECHO(S) DA(S) RODOVIA(S): analisar os dados consultados somente ao(s) trecho(s) escolhido(s);

4. TRECHO(S) DA(S) RODOVIA(S) E CONDUTOR(ES): analisar os dados referentes ao seu perfil de condutor de acordo com o(s) trecho(s); 
5. TRECHO(S) DA(S) RODOVIA(S) E VEÍCULO(S): analisar os dados que relacionam o(s) trecho(s) e o tipo de veículo escolhido;

6. TRECHO(S) DA(S) RODOVIA(S) E CONDUTOR(ES) E VEÍCULO(S): analisar os dados especificos para o perfil de condutor e tipo de veículo escolhido dentro do(s) trecho(s) determinado(s).
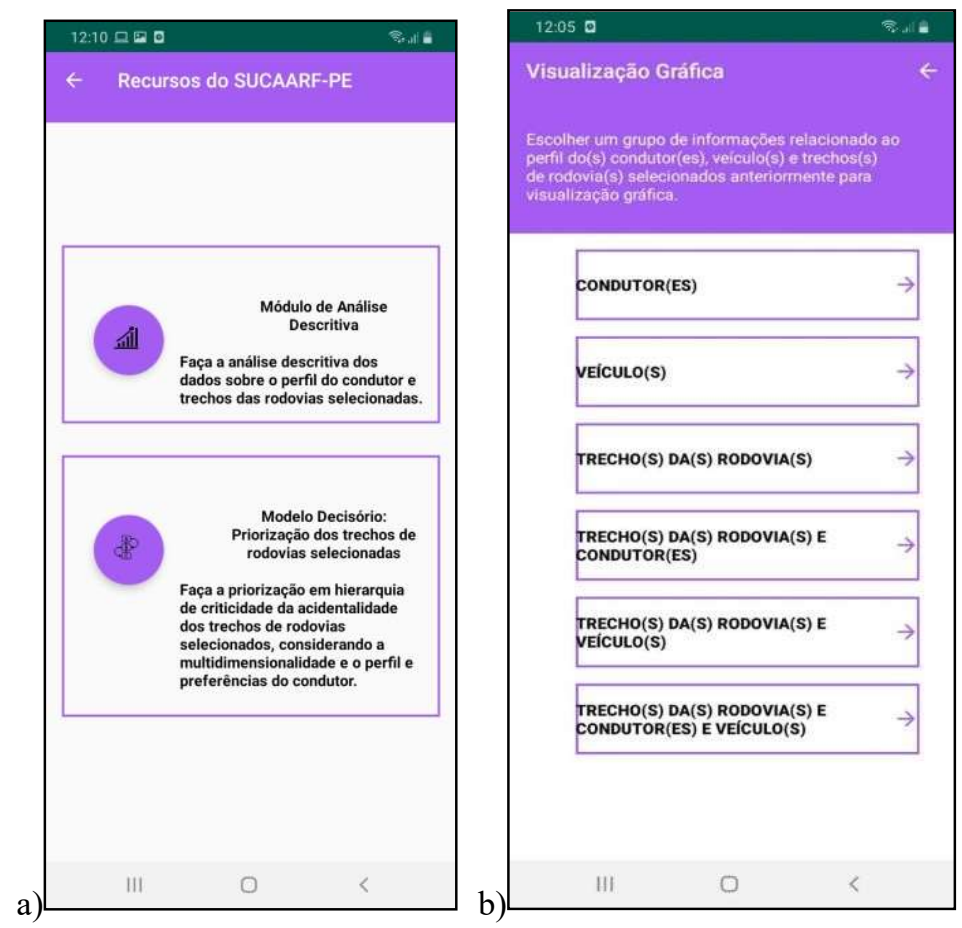

Figura 4 - Interfaces de entrada de dados do SAD SUCAARF-PE: a) Módulos disponíveis no SUCAARF-PE; b) Opções de análise dentro do módulo de análise descritiva)

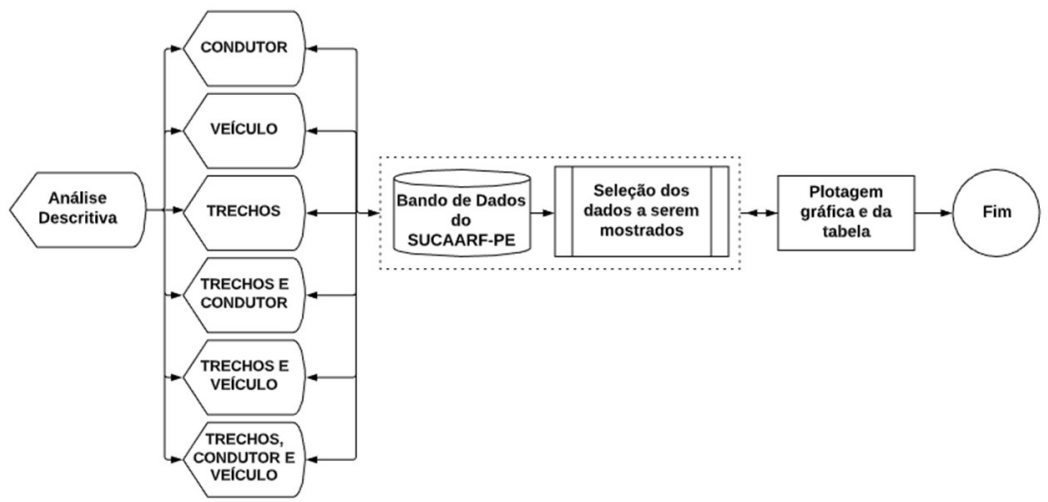

Figura 5 - Esquema do módulo de análise descritiva.

Para cada opção de análise escolhida é construído um gráfico e uma tabela de leitura onde o usuário visualiza e analisa as informações de forma facilitada sobre as circunstâncias que resultaram os acidentes, sendo composto pelas variáveis: i) Causas do Acidente, ii) Estado Físico dos envolvidos; e iii) Tipos de Acidente. A Figura 6 apresenta a estrutura das seis interfaces de análise descritiva disponíveis neste módulo. 

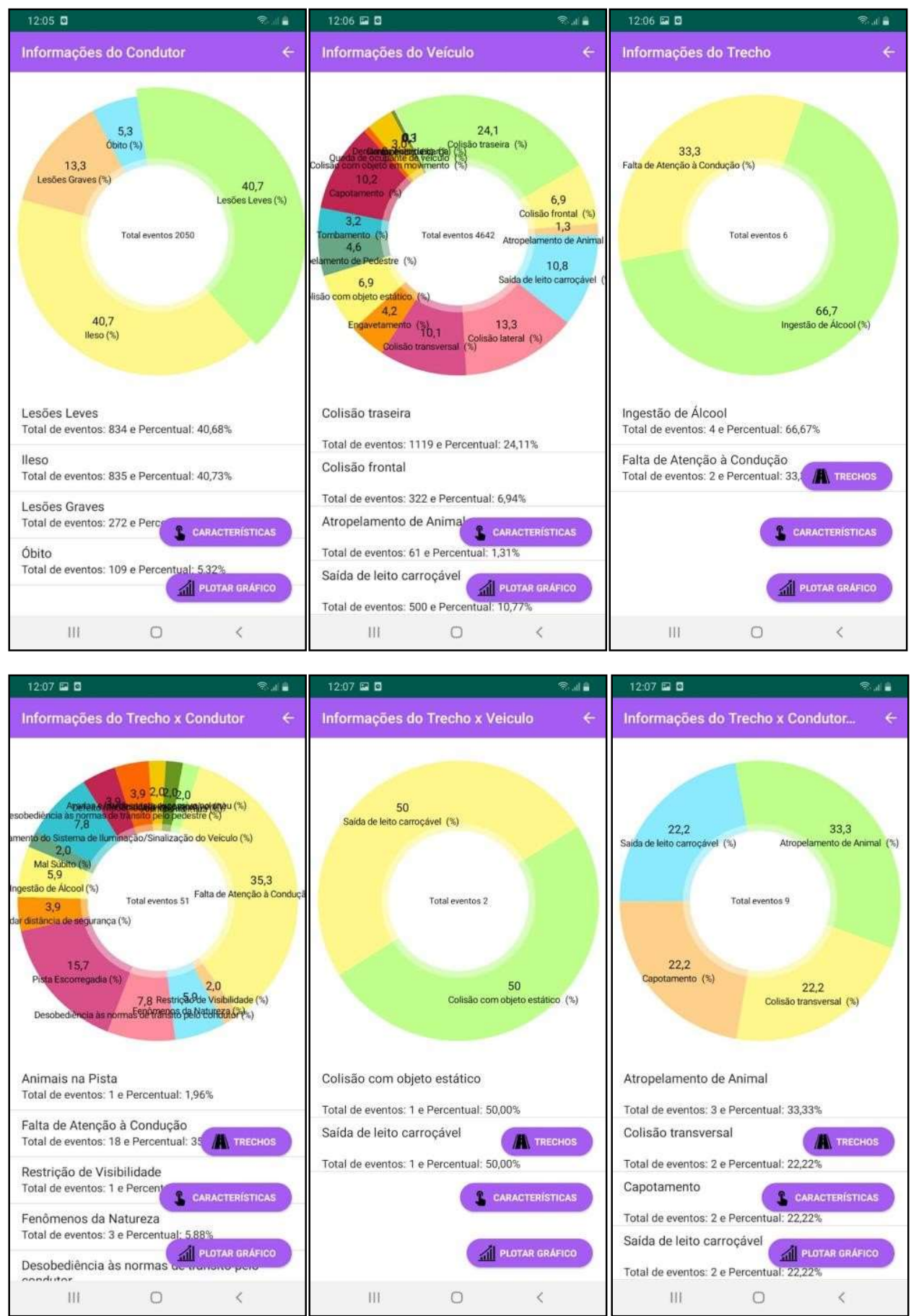

Figura 6 - Telas interativas de visualização das informações gráficas e tabular do módulo de análise descritiva.

Para a estruturação de uma aplicação prática do módulo de análise descritiva, e posteriormente do módulo do modelo decisório, será considerado que um usuário (motorista) defina um perfil de condutor no SUCAARF-PE com os seguintes dados: genêro "Masculino", faixa etária "Até 29 anos de idade", tipo de veículo "Automóvel” e uma rota de percurso abrangendo os trechos presentes na Tabela 3.

Através do módulo de análise descritiva e das informações obtidas pelas interfaces de análise apresentadas, é possível evidenciar, considerando os dados do perfil de condutor definido anteriormente, que, por exemplo:

- Os acidentes envolvendo o genêro "Masculino" e faixa etária "Até 29 anos de idade" resultam 
estatisticamente em 40.73\% em "Ilesos" e 40.68\% em "Lesões Leves", como os estados fisícos dos envolvidos.

- Os acidentes envolvendo o tipo de veículo "Automóvel" resultam em 24.11\% em "Colisão traseira", como o principal tipo de acidente.

- Os acidentes ocorridos no trecho "BR101/PE_0_7,7km" (primeiro trecho da Tabela 3) resultam em 66.67\% em "Ingestão de álcool”, como a principal causa dos acidentes.

\subsection{Modelo de decisão para priorização dos trechos das rodovias sob a abordagem multicritério}

O modelo de decisão multicritério do SUCAARF-PE tem como objetivo a priorização dos trechos das rodovias sob a abordagem multicritério, na qual auxiliará no processo de decisão considerando múltiplos critérios de forma integrada e agregando as preferências do decisor (DE ALMEIDA, 2013). Para isso, algumas etapas do processo de tomada de decisão são propostas, conforme mostrada na Figura 7 e detalhados na Tabela 1.

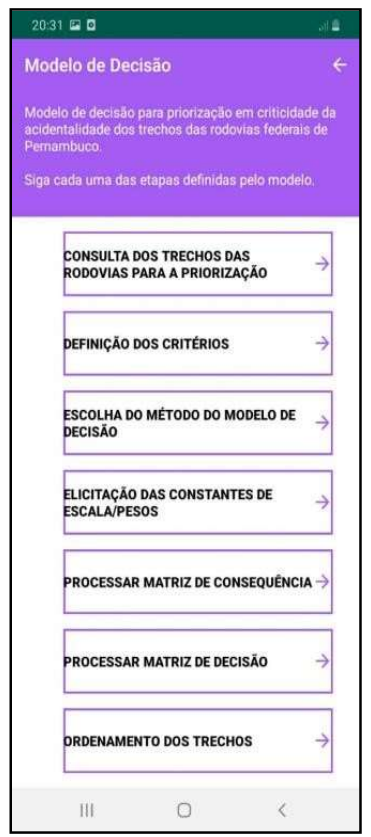

Figura 7 - Etapas do modelo decisório de priorização do SUCAARF-PE

Neste problema de decisão, as alternativas $\left(x_{i}\right)$ a serem avaliadas são os trechos de rodovias de interesse de análise do decisor dentro do sistema de rodovias federais de Pernambuco. Já quanto aos critérios $\left(c_{j}\right)$, tem-se um conjunto pré-cadastrado de oito critérios, conforme identificados por Martins (2020), que serão extraídos da base de dados da PRF. No entanto, caso o decisor sinta-se na necessidade de inserir novos critérios é preciso inserir os dados desse critério no banco de dados (a ser desenvolvido). Os oitos critérios $\left(c_{j}\right)$ considerados são:

- Índice de Acidentes $\left(c_{1}\right)$ : valor absoluto de ocorrências registradas (acidentes) na alternativa $x_{i}$;

- Índice de Ilesos $\left(c_{2}\right)$ : índice de pessoas ilesas (acidentes os quais possuem apenas danos materiais e consequências não físicas a vítima) na alternativa $x_{i}$;

- $\quad$ Índice de Lesões Leves $\left(c_{3}\right)$ : índice de pessoas feridas levemente em acidentes na alternativa $x_{i}$;

- Índice de Lesões Graves $\left(c_{4}\right)$ : índice de pessoas feridas gravemente em acidentes na alternativa $x_{i}$;

- Índice de Mortos $\left(c_{5}\right)$ : índice de pessoas mortas em acidentes na alternativa $x_{i}$;

- Qualidade do Pavimento $\left(c_{6}\right)$ : característica qualitativa do pavimento da alternativa $x i$, sendo considerado condições de superfície e pontos críticos;

- Qualidade da Geometria $\left(c_{7}\right)$ : característica qualitativa da geométrica da alternativa $x i$, sendo considerado perfil da rodovia, presença de curvas perigosas, presença de acostamento;

- Qualidade da Sinalização $\left(c_{8}\right)$ : característica qualitativa da sinalização da alternativa $x i$, sendo considerado presença e condições da sinalização, presença de placas de velocidade, sua visibilidade e legibilidade. 


\begin{tabular}{|c|c|}
\hline Etapas & Objetivo \\
\hline $\begin{array}{l}\text { Consulta dos trechos das rodovias } \\
\text { para a priorização }\end{array}$ & $\begin{array}{l}\text { Permitir visualizar os trechos selecionados no módulo de Definição do } \\
\text { Perfil }\end{array}$ \\
\hline Definição dos critérios & $\begin{array}{l}\text { Permitir a seleção dos critérios mais críticos para o usuário, dentre os oito } \\
\text { critérios de Martins (2020) }\end{array}$ \\
\hline $\begin{array}{l}\text { Escolha do método do modelo de } \\
\text { decisão }\end{array}$ & $\begin{array}{l}\text { Definir o modelo de decisão através da racionalidade compensatória } \\
\text { (modelo de agregação aditiva) ou não compensatória (PROMETHEE) do } \\
\text { usuário }\end{array}$ \\
\hline $\begin{array}{l}\text { Elicitação das constantes de } \\
\text { escala/pesos }\end{array}$ & $\begin{array}{l}\text { Definir o método de obtenção dos pesos dos critérios selecionados, } \\
\text { definição manual ou método Rank-Order Centroid - ROC }\end{array}$ \\
\hline Processar matriz de consequências & Levantamento do desempenho das alternativas nos diferentes critérios \\
\hline Processar matriz de decisão & $\begin{array}{l}\text { Desempenho normalizado da avaliação intra-critério, de cada alternativa } \\
\text { para cada critério }\end{array}$ \\
\hline Ordenamento dos trechos & $\begin{array}{l}\text { Priorização (ordenamento) dos trechos selecionados, através da função } \\
\text { valor v(x), em uma hierarquia de criticidade de acidentes de trânsito }\end{array}$ \\
\hline
\end{tabular}

Tabela 1 - Etapas do modelo de priorização das rodovias federais de Pernambuco

Os dados utilizados para os critérios qualitativos das alternativas, representados pela qualidade do pavimento, qualidade da geometria e qualidade da sinalização, foram retiradas dos relatórios gerencias do Conselho Nacional de Trânsito (CNT). Segundo o relatório de 2018 (CNT, 2018) e, utilizando uma conversão de escala (4: Péssimo, 3: Ruim, 2: Regular, 1: Bom, 0: Ótimo), obtiveram os desempenhos conforme mostrados na Tabela 2.

\begin{tabular}{c|c|c|c|c|c|c}
\hline \multirow{2}{*}{$\begin{array}{c}\text { Trechos de } \\
\text { rodovias analisadas }\end{array}$} & \multicolumn{2}{c|}{ Qualidade do Pavimento } & \multicolumn{2}{c|}{ Qualidade da Geometria } & \multicolumn{2}{c}{ Qualidade da Sinalização } \\
\cline { 2 - 8 } & Desempenho & Escala & Desempenho & Escala & Desempenho & Escala \\
\hline BR101 & Regular & 2 & Regular & 2 & Regular & 2 \\
\hline BR104 & Bom & 1 & Ruim & 3 & Regular & 2 \\
\hline BR110 & Bom & 1 & Ruim & 3 & Regular & 2 \\
\hline BR116 & Bom & 1 & Ruim & 3 & Regular & 2 \\
\hline
\end{tabular}

Tabela 2 - Qualificações dos critérios qualitativos das rodovias e escala correspondete utilizada

Fonte: adaptado do relatório do CNT (CNT, 2018).

Posteriormente, é preciso identificar as características de preferência do decisor (usuário) com o objetivo de escolher o modelo de decisão mais adequado ao perfil do decisor. Para isso, algumas perguntas básicas serão respondidas pelo decisor afim de identificar a racionalidade compensatório ou não do decisor. A depender da racionalidade identificada do usuário, o SUCAARF-PE irá encaminhar automaticamente o decisor para o modelo mais adequado, que seria o método de agregação aditiva para o caso de racionalidade compensatória ou o método de sobreclassificação (especificamente, o método PROMETHEE II) para a racionalidade não-compensatória.

Uma vez identificado e escolhidos os critérios e o modelo de agregação multicritério mais adequado, é preciso elicitar do decisor as constantes de escala (ou pesos) $\left(w_{j}\right)$ para cada critério. Para tanto, o SAD disponibiliza, como forma auxiliar, o procedimento de Rank-Order Centroid (ROC) (BARRON; BARRETT, 1996) para elicitar as constantes de escala (ou pesos), conforme dado pela Equação 1. Escolheu-se, inicialmente, o método ROC como umas das ferramentas disponíveis pelo SAD, pois ele exige informação parcial do decisor (através da definição do ordenamento dos critérios) e, consequentemente, requer menor esforço do decisor, conforme exemplo da Figura 8.

Além disso, o SUCAARF-PE deixa a possibilidade do decisor inserir manualmente os valores das constantes de 
escala (ou pesos). Isto permite uma maior flexibilidade do decisor poder usar outra metodologia que seja mais adequado ou conveniente a sua racionalidade e esforço cognitivo.

$$
\mathrm{w}_{j}=\frac{1}{n_{i}} \sum_{\mathrm{j}=1}^{\mathrm{n}_{\mathrm{i}}} \frac{1}{\mathrm{j}}
$$

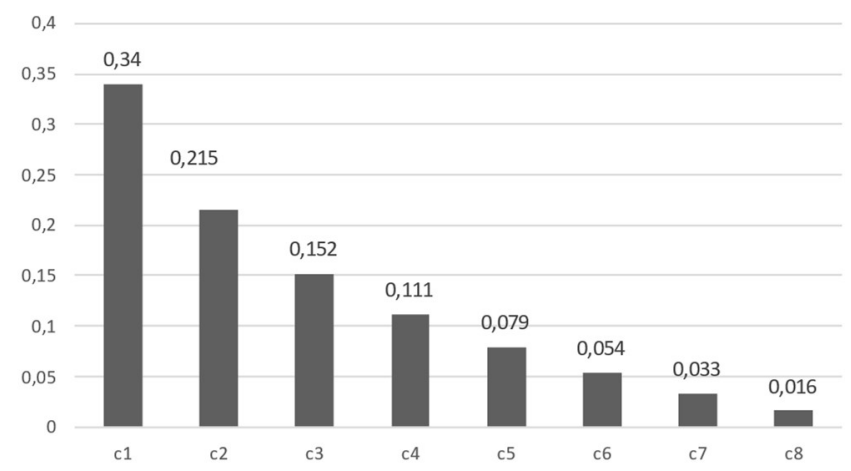

Figura 8 - Aplicação do método ROC conforme ordenação descrescente de preferência dos critérios

Em seguida, com base em cada trecho de rodovia $x_{i}$ considerado e para cada critério $j$, é feita a avaliação intracritério, a qual estima-se à função valor $v_{j}\left(x_{i}\right)$, conforme mostrada na Tabela 3 .

\begin{tabular}{|c|c|c|c|c|c|c|c|c|c|c|}
\hline \multirow[b]{3}{*}{ Trechos } & \multicolumn{8}{|c|}{ Pesos } & \multirow[b]{3}{*}{$v\left(x_{i}\right)$} & \multirow[b]{3}{*}{ Priorização } \\
\hline & $c_{1}$ & $c_{2}$ & $c_{3}$ & $c_{4}$ & $c_{5}$ & $c_{6}$ & $c_{7}$ & $c_{8}$ & & \\
\hline & 0,340 & 0,215 & 0,152 & 0,111 & 0,079 & 0,054 & 0,033 & 0,016 & & \\
\hline BR101/PE_0_7,7km & 0,759 & 0,741 & 0,692 & 0,829 & 0,208 & 1 & 1 & 0 & 0,720 & $2^{\circ}$. \\
\hline BR101/PE_0_11,3km & 1 & 1 & 0,885 & 1 & 0,542 & 1 & 1 & 0 & 0,930 & $1^{\circ}$ \\
\hline BR104/PE_0_12km & 0,007 & 0 & 0,038 & 0,010 & 0,083 & 0 & 1 & 1 & 0,065 & $6^{\circ}$ \\
\hline BR104/PE_67,9_71,2km & 0,227 & 0,151 & 0,270 & 0,267 & 0,375 & 0 & 1 & 1 & 0,259 & $5^{\circ}$ \\
\hline BR110/PE_94,3_148km & 0,345 & 0,144 & 0,577 & 0,371 & 1 & 0 & 1 & 1 & 0,405 & $4^{\circ}$ \\
\hline BR110/PE_219,6_225,7km & 0 & 0,007 & 0 & 0,010 & 0 & 0 & 1 & 1 & 0,051 & $8^{\circ}$ \\
\hline BR116/PE_25,7_43,5km & 0,503 & 0,260 & 1 & 0,705 & 0,250 & 0 & 1 & 1 & 0,525 & $3^{\circ}$ \\
\hline BR116/PE_82,7_91,6km & 0,003 & 0,014 & 0 & 0 & 0,042 & 0 & 1 & 1 & 0,057 & $7^{\circ}$ \\
\hline
\end{tabular}

Tabela 3 - Matriz de decisão - exemplo usando o modelo de agregação aditiva

Posteriormente, realiza-se a agregação multicritério através da agregação aditiva (dado que é considerado que a racionalidade do usuário (decisor) é compensatória), dada pela Equação (2). Por fim, com todos os valores $v\left(x_{i}\right)$ calculados, ordena-se as alternativas numa hierarquia de criticidade da acidentalidade dos trechos de rodovias analisadas.

$$
v\left(x_{i}\right)=\sum_{j=1}^{n} w_{j} v_{j}\left(x_{i}\right)
$$

onde, $n$ é a quantidade de critérios definidos e $\sum_{j=1}^{n} w_{j}=1$.

Para a apresentação ao usuário do SUCAARF-PE, supondo que o usuário defina os oito critérios disponibilizados como sendo relevantes a serem considerados, sendo definido como racionalidade compensatória (modelo de agregação aditiva) e utilizando o método de obtenção dos pesos dos critérios, o ROC, o módulo decisório apresenta 
como resultado o ordenamento dos trechos selecionados em hierarquia de criticidade (trecho mais crítico ao menos crítico), sendo o trecho 'BR101/PE_0_11,3km' o mais crítico nesta situação, conforme mostra a Figura 9.

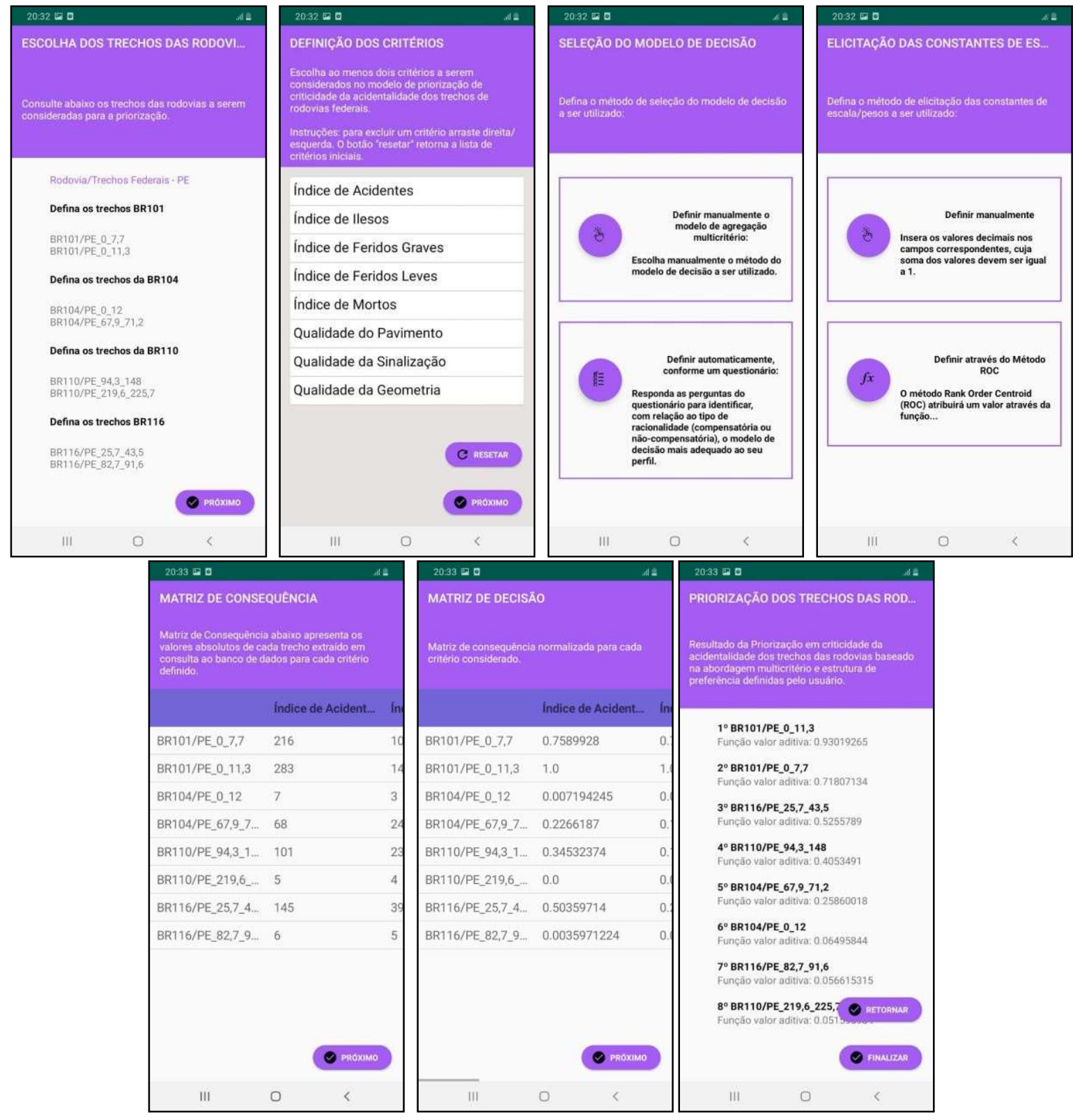

Figura 9 - Aplicação do modelo de decisão para priorização

\section{Discussões}

Como forma de demonstrar os diferentes resultados obtidos pelos recursos do SUCAARF-PE para diferentes tipos de perfis de condutor, e ademais, possibilitar enriquecer o trabalho trazendo conteúdo para discussão dos resultados e propostas de melhorias na implementação realizada, será implementado um estudo comparativo dos resultados da ferramenta para dois perfis de condutor que realizarão um trajeto percorrendo os trechos $A_{1}: \quad$ BR104/PE_0_12_43,5km; $\quad A_{2}: \quad$ BR104/PE_30,5_31 km; $\quad A_{3}: \quad$ BR104/PE_67,9_71,2km; $A_{4}:$ BR104/PE $87,9 \_106,1 \mathrm{~km}$.

- Usuário 1: genêro "Feminino", faixa etária "Até 29 anos de idade" e tipo do veículo "Automóvel".

- Usuário 2: genêro "Masculino", faixa etária "30 a 59 anos" e tipo do veículo "Caminhão".

O primeiro recurso implementado da ferramenta será o módulo de Análise Descritiva, onde para cada uma das seis 
opções de análises distintas, são verificados os valores percentuais das causas, estados físicos e tipos de acidentes à cada perfil, como mostrado na Tabela 4 para ambos os usuários, sendo evidenciados os três primeiros módulos de análise e as principais caracteristicas obtidas.

\begin{tabular}{|c|c|c|c|}
\hline & Causas do Acidente & Estado Físico dos envolvidos & Tipos de Acidente \\
\hline \multicolumn{4}{|c|}{ Perfil do Usuário 1} \\
\hline Condutor(es) & 35,3\% em "Falta de Atenção à Condução" & 54,7\% em "Lesões Leves" & $16 \%$ em "Colisão Traserira" \\
\hline Veículo(s) & 40,1\% em "Falta de Atenção à Condução" & $63,7 \%$ em "Ilesos" & 24,1\% em "Colisão Traserira" \\
\hline$A_{1}$ & 71,43\% em "Falta de Atenção à Condução" & $42,86 \%$ em "Ilesos" & 42,86\% em "Colisão Transversal" \\
\hline$A_{2}$ & $60 \%$ em "Ultrapassagem Indevida" & $70 \%$ em "Ilesos" & $40 \%$ em "Tombamento" \\
\hline$A_{3}$ & 23,5\% em "Falta de Atenção à Condução" & 41,2\% em "Lesões Leves" & 17,6\% em "Colisão Traseira" \\
\hline$A_{4}$ & 24,7\% em "Falta de Atenção à Condução" & 46,8\% em "Lesões Leves" & 27,3\% em "Colisão Frontal" \\
\hline \multicolumn{4}{|c|}{ Perfil do Usuário 2} \\
\hline Condutor(es) & 39,3\% em "Falta de Atenção à Condução" & $54,6 \%$ em "Ilesos" & 22,9\% em “Colisão Traserira" \\
\hline Veículo(s) & 36,9\% em "Falta de Atenção à Condução" & $67,9 \%$ em "Ilesos" & 25\% em "Colisão Traserira" \\
\hline$A_{1}$ & 71,43\% em "Falta de Atenção à Condução" & $42,86 \%$ em "Ilesos" & 42,86\% em "Colisão Transversal" \\
\hline$A_{2}$ & $60 \%$ em "Ultrapassagem Indevida" & $70 \%$ em "Ilesos" & $40 \%$ em "Tombamento" \\
\hline$A_{3}$ & 23,5\% em "Falta de Atenção à Condução" & $41,2 \%$ em "Lesões Leves" & 17,6\% em "Colisão Traseira" \\
\hline$A_{4}$ & 24,7\% em "Falta de Atenção à Condução" & 46,8\% em "Lesões Leves" & 27,3\% em "Colisão Frontal" \\
\hline
\end{tabular}

Tabela 4 - Módulo de Análise Descritiva- exemplo usando diferentes perfis de condutor

Posteriormente, o segundo recurso oferecido pela ferramenta sendo o Módulo do Modelo Decisório, onde é considerado nesse momento as alternativas (trechos definidos anteriormente) e os critérios. Para as escolhas dos critérios, o Usuário 1 considerou os oitos critérios disponíveis no SAD, e o Usuário 2 considerou somente os cincos critérios quantitativos. Além disso, foi considerado que os dois usuários possuem uma racionalidade compensatória (ou seja, utilizaram o modelo de agregação aditiva) e utilizaram o método para a obtenção dos pesos dos critérios através do método ROC. Para a aplicação do ROC, a ordem de preferência dos critérios definidas pelos usuários foram: Usuário 1 sendo $c_{1}>c_{2}>c_{3}>c_{4}>c_{5}>c_{6}>c_{7}>c_{8}$; e para o Usuário 2 sendo $c_{2}>c_{5}>c_{4}>c_{3}>$ $c_{1}$. Portanto, os pesos obtidos pelo método ROC (Equação 1) são mostrados na Tabela 5.

Determinado os pesos e agregando os múltiplos critério de forma aditiva (Equação 2), tem-se resultado do modelo decisório, que é o ordenamento dos trechos selecionados em hierarquia de criticidade, conforme mostrado na Figura 10. Os trechos das rodovias que os usuários no exemplo susposto devem deter uma maior atenção durante o seu trajeto, são respectivamente os trechos "BR104/PE_87,9_106,1km" e "BR104/PE_67,9_71,2km" para o Usuário 1 e Usuário 2, respectivamente.

\begin{tabular}{lcccccccc}
\hline & \multicolumn{10}{c}{$\boldsymbol{w}_{\boldsymbol{j}}$} \\
\cline { 2 - 9 } Usuário & $\boldsymbol{c}_{\boldsymbol{1}}$ & $\boldsymbol{c}_{\boldsymbol{2}}$ & $\boldsymbol{c}_{\boldsymbol{3}}$ & $\boldsymbol{c}_{\boldsymbol{4}}$ & $\boldsymbol{c}_{\mathbf{5}}$ & $\boldsymbol{c}_{\boldsymbol{6}}$ & $\boldsymbol{c}_{\mathbf{7}}$ & $\boldsymbol{c}_{\boldsymbol{8}}$ \\
\hline Usuário 1 & 0,334 & 0,215 & 0,152 & 0,111 & 0,079 & 0,054 & 0,033 & 0,016 \\
Usuário 2 & 0,040 & 0,456 & 0,090 & 0,156 & 0,256 & - & - & - \\
\hline
\end{tabular}

Tabela 5 - Ordenamento e peso dos critérios obtidas pelo método ROC

Portanto, é perceptível que diferentes fatores influênciam e individualizam os resultados de ordenamento da criticidade dos trechos obtidos pelo modelo de decisão, pois a criticidade de uma via está relacionada a diferentes fatores de risco, tais como, perfil do condutor considerado para a análise, critérios de impacto considerados pelo usuário, a ordem de criticidade dos critérios escolhidos, o tipo de racionalidade do decisor, etc. 

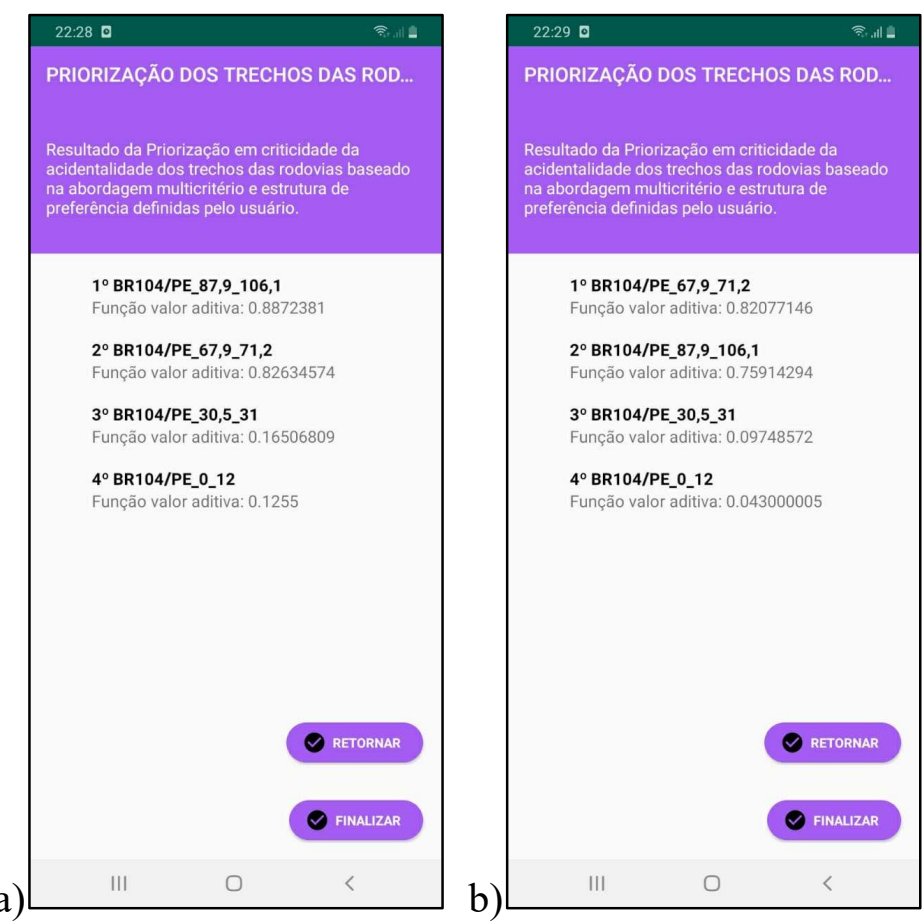

Figura 10 - Ordenamentos em ordem de criticidade das alternativas a cada usuário: a) Usuário 1; b) Usuário 2.

\section{Conclusões}

O trabalho teve como objetivo principal apresentar um sistema de apoio à decisão (SAD), chamado de SUCAARF$\mathrm{PE}$, que foi desenvolvido para auxiliar e embasar o processo de tomada de decisão de usuários de rodovias federais (motoristas em geral), e geração de conhecimento através da disponibilização de informações eficientes acerca das acidentalidades nas rodovias federais do estado de Pernambuco.

As principais contribuições do artigo podem ser resumidas em: i) o SUCAARF-PE é, dentro do nosso conhecimento, o primeiro de seu tipo, ou seja, incorporando as preferências individuais do decisor (motorista) e dados históricos de acidentes em rodovias, em um estudo de avaliação e priorização de trechos (rota de percurso do usuário), como também, determinação dos trechos de maior atenção na rota; ii) o aplicativo possui capacidade de ser disponibilizado e obtido gratuitamente aos usuários, composto pelos atores diretos das rodovias federais pernambucanas (motoristas), permitindo o acesso remoto as informações, sendo necessário apenas um smartphone Android; e, iii) devido à integração e obtenção de dados de acidentes em rodovias federais comumente coletados, o SAD proposto pode ser facilmente estendido a outras partes do mundo onde esses dados são compilados, em seus respectivos níveis regionais ou nacionais.

Sendo assim, os principais resultados obtidos pelos usuários de rodovias federais ao uso do SUCAARF-PE são: i) disponibilização de uma análise descritiva dos dados através de informações gráficas e tabelas de leituras referentes a um perfil de analise definido pelo usuário; ii) disponibilização de um modelo de decisão multicritério de priorização e ordenamento das alternativas (trechos) de acordo com a definição dos critérios de maior criticidade do decisor; iii) possibilitar através do resultado do modelo decisório, suporte a tomada de decisão aos usuários de rodovias através da avaliação dos trechos aos quais irão compor a rota de percurso do decisor, como também avaliação dos trechos de maior atenção (criticidade) durante o percurso.

Como trabalho futuro, sugerimos realizar um experimento controlado com usuários de diferentes perfis para que a anáise de feedback possa enriquecer possíveis melhorias na implementação do SAD proposto, para então, ser disponibilizado em mercado aberto. Além disso, adaptar o código de programação afim de atender outros tipos de usuários além da plataforma Android.

\section{Agradecimentos}

Este trabalho foi parcialmente apoiado pela Coordenação de Aperfeiçoamento de Pessoal de Nível Superior 
(CAPES) e pelo Conselho Nacional de Desenvolvimento Científico e Tecnológico (CNPq) (407541/2018-7).

Referências

BARRON, F. H.; BARRETT, B. E. The efficacy of SMARTER - Simple Multi-Attribute Rating Technique Extended to Ranking. Acta Psychologica, v. 93, n. 1-3, p. 23-36, set. 1996.

CNT. Confederação Nacional dos Transportes - Pesquisa CNT de Rodovias 2018. Disponível em: $<$ https://pesquisarodovias.cnt.org.br/Downloads/Edicoes//2018/Relatório Gerencial/Pesquisa CNT (2018) ALTA.pdf $>$. Acesso em: 1 fev. 2020.

DE ALMEIDA, A. T. Processo de Decisão nas Organizações: Construindo Modelos de Decisão Multicritério (Decision Process in Organizaions: Building Multicriteria Decision Models). 1. ed. São Paulo/SP, Brasil: Editora Atlas, 2013.

DE ALMEIDA, A. T. et al. A new method for elicitation of criteria weights in additive models: Flexible and interactive tradeoff. European Journal of Operational Research, v. 250, n. 1, p. 179-191, abr. 2016.

DE ALMEIDA, A. T.; RAMOS, F. S. Org. Gestão da Informação na competitividade das organizações. 3a Edição ed. Recife: Editora Universitária UFPE, 2002.

FREJ, E. A.; DE ALMEIDA, A. T.; COSTA, A. P. C. S. Using data visualization for ranking alternatives with partial information and interactive tradeoff elicitation. Operational Research, v. 19, n. 4, p. 909-931, 3 dez. 2019.

MARTINS, M. A. Modelo de decisão para redução de risco de acidentes de trânsito nas rodovias federais de Pernambuco: desenvolvimento de um sistema de apoio à decisão. [s.1.] (dissertação) Universidade Federal de Pernambuco, CAA, Programa de Pós-Graduação de Engenharia de Produção, 2020.

MARTINS, M. A. et al. Multicriteria Model Based on FITradeoff Method for Prioritizing Sections of Brazilian Roads by Criticality. Mathematical Problems in Engineering, v. 2020, p. 1-15, 29 dez. 2020.

MARTINS, M. A.; GARCEZ, T. V. Análise descritiva dos acidentes nas rodovias federais de Pernambuco (2007-2015). XXXVII Encontro Nacional de Engenharia de Produção. Anais...Joinville/SC: Associação Brasileira de Engenharia de Produção, 2017

MARTINS, M. A.; GARCEZ, T. V. Desenvolvimento de um Sistema de Informação para Acidentes de Trânsito Ocorridos em Pernambuco. Gestão.Org, v. 17, n. EE, p. 149-162, 30 out. 2019.

PEARSON, J. M.; SHIM, J. P. An empirical investigation into decision support systems capabilities: A proposed taxonomy. [s.l.] Information \& Management, 1994.

RATHNAM, S.; MANNINO, M. V. Tools for building the human-computer interface of a decision support system. [s.1.] Decision Support Systems, 1995.

RYDER, B. et al. Preventing traffic accidents with in-vehicle decision support systems - The impact of accident hotspot warnings on driver behaviour. Decision Support Systems, v. 99, p. 64-74, jul. 2017.

SHIM, J. P. Past, present, and future of decision support technology. [s.1.] Decision Support Systems, 2002.

SPRAGUE, J.; WATSON, H. J. Sistema de apoio à decisão: Colocando a teoria em prática. Rio de Janeiro: Campus, 1991.

STAIR, R. M.; REYNOLDS, G. W.; DA SILVA, F. S. C. Princípios de sistemas de informação. 9. ed. São Paulo: Cengage Learning, 2011.

WHO. World Health Organization. Road traffic injuries. Geneva: [s.n.]. 